\title{
Constructing a caring training content for nurse managers: a Delphi study
}

Original article

Rui Chenª, Yi-Lan Liub,*

${ }^{a}$ Department of Nursing Administration, 'Luoyang Orthopedic Hospital of Henan Province, Orthopedic Hospital of Henan Province, Zhengzhou, Henan 450000, China

${ }^{b}$ Department of Nursing, Union Hospital of Tongji Medical College, Huazhong University of Science and Technology, Wuhan, Hubei 430000, China

Received: 27 June 2018; Accepted: 9 September 2018; Published: 20 June 2019

Abstract: Objective: To design a scientific, feasible caring training content for nurse managers.

Methods: Training content came from review of literature; all the content were sorted out, analyzed, and compiled by the research team. Then, a Delphi method was used to collect opinions from experts on the caring training contents.

Results: A 22-member multidisciplinary panel was engaged in a two-round Delphi process to assess the importance of the items and to identify any additional elements. Consensus was obtained on 23 items that belonged to four modules. There were 10 items related to caring knowledge and caring ability, six items related to caring leadership, three items related to caring education, and four items related to caring research.

Conclusions: The four-module integrated training contents achieved in this study represented the core caring knowledge and skills that a nurse manager who worked in hospital needed to know. Nurse managers can choose any module to have a system learning according to their practical needs, totally or separately.

Keywords: caring • training $\bullet$ nurse manager $\bullet$ Delphi technique

(c) Shanxi Medical Periodical Press.

\section{Introduction}

Caring is often considered a synonym for nursing. Interest in caring has grown exponentially from nursing theorists, researchers, clinicians, and educators. ${ }^{1}$ Caring has been directly related to quality of care, healthcare economics, patient outcomes, administration practices, and nurse and patient satisfaction. ${ }^{2-7}$ Nurse mangers play a pivotal role in nursing care, and they are responsible for promoting efficient, effective, safe, and compassionate nursing care. ${ }^{8}$ Nurse managers are in positions between doctors and nurses, between hospital leaders and nurses, and between other nurses. They have been at the top, the bottom, and in between, where they must make appropriate decisions while continuing their nursing management. ${ }^{2,9}$ Nurse managers have sufficient power and organizational influence to obtain the resources necessary to promote nursing caring practices. ${ }^{10,11}$ Leininger ${ }^{12}$ believed that nurse managers and

How to cite this article: Chen R, Liu Y-L. Constructing a caring training content for nurse managers: a Delphi study. Front Nurs. 2019; 2: 1-8.

O Open Access. (c) 2019 Rui Chen and Yi-Lan Liu, published by Sciendo. (cc) BY-NC-ND This work is licensed under the Creative Commons Attribution NonCommercial-NoDerivatives 4.0 License. 
administrators can embody caring and improve patient care by insisting on a caring philosophy from top to bottom within the nursing service. Results from phenomenological study ${ }^{10,13}$ also revealed that staff nurses experienced difficulties in caring for their patients when they, themselves, have not felt cared for or appreciated by their nurse manager. Similarly, quantitative research of Wade et al. ${ }^{14}$ also showed that caring attributes of managers was a statistically significant predictor of nurses' job enjoyment. Thus, some authors argued that nurse managers needed to have enough caring knowledge, caring ability, and caring skills to make sure that they can better portray their role, care for their staff, take care of their patients and families, and also enhance the organization culture. ${ }^{15-17}$

It is regretful that nurse mangers are faced with the dilemma of how to take good care of their firstline nurses, 2,17,18 and there are also evidences in the recent literature that clinical nurses consider that they do not generally get enough support from their nurse managers. ${ }^{13,19-24}$ It prompted us to explore a practical and effective way to solve this problem. In a previous study, we also explored the caring training experiences and expectation of 453 nurse managers in 14 hospitals in the Hubei Province in China. Results showed that $96.9 \%$ nurse managers need caring training and $37.7 \%$ nurse managers have not been trained in the caring field. Although it is widely accepted that a caring training is integral to nursing continuing education, ${ }^{6,25,26}$ there is an apparent dearth of a standardized caring training for nurse managers. It is urgent to develop consistent training content for nurse managers to acquire caring knowledge, improve caring ability and skills, inspire caring behavior, enhance the organization climate, and improve nurse and patient satisfaction.

\section{Literature review}

Caring in education has been emphasized as the first place for teaching and learning the values of the nursing profession. ${ }^{27}$ In the past several decades, caring in nursing education was conspicuously identified and adopted by schools and hospitals. Many countries initiated the efforts, such as Canada, Japan, and China, especially the United States. Many organizations in America made great efforts on developing nursing caring education. A literature review revealed that the majority of nursing caring education programs were based on Waston's theory, and grounded in American, they could be classified into four groups according to the target audience: most of them focused on nursing students, ${ }^{25,28-34} \mathrm{sev}$ eral of them focused on clinical nurses, ${ }^{26,35-39}$ and a few of them focused on nursing educators ${ }^{40,41}$ and nursing administrators. ${ }^{42}$ Some paradigms exist. The Weber
State University, USA, offers a BSN course focused on Jean Watson's definition of caring. It includes caring for self, others, peers/co-workers, nursing leadership, local/ world communities, the environment, and Web-based interactions. ${ }^{28}$ The course created for nurse educators at Inova Alexandria Hospital ${ }^{41}$ was a noteworthy exception. It not only focused on offering a caring training for nurse educators to help them influence nurses in their wards but also had a continuing caring training last along for 3 years to make sure that affective growth occurred for both clinicians and educators. Implementation included core content in a 30-minute training session, large-scale leadership conferences, and consistent educator support (pilot units in patient areas and outpatient areas). Nursing researchers also had described some theoretical aspects of caring education. More and more scholars were committed to develop the online courses and video courses. ${ }^{43}$ Through the literature review, it was not difficult to find that most literature concentrated on caring education for nursing students and clinic nurses, with little description of the education program for nurse managers. Summarizing the caring program content in nursing education, we found that caring education was a gradual, systematic, and continuous process that causes the caring education effectual. It was regrettable that still no literature identified what content and skills should be included in caring training content for nurse managers.

\section{Methods}

\subsection{Development of the questionnaire}

We preliminarily drafted the questionnaire, which contained three parts: (1) a cover letter detailing the aim and background of this study; (2) a demographic questionnaire including gender, age, degree, years of experience, position, and discipline; and (3) a questionnaire, which was the preliminary designed training program from literature review and the cross-sectional study. ${ }^{44}$ Participants were asked to rate the importance of each item that was regarded as an essential component using a 5-point Likert-type scale (I=not at all important, $2=$ not very important, $3=$ neutral, $4=$ fairly important, and $5=$ very important). If there was any disagreement ( 1 or 2), experts should give their comments. Additional space was provided within the tool for participants to suggest items that they felt were important but not contained in the tool.

Prior to mailing the surveys to participants, five experts in the related field had finished the pilot tested for feasibility and content validity. Their suggestions were related to wording changes intended to increase 
the clarity of the questionnaire. Based on the results of the pilot, minor changes were made to the format of the questionnaire.

\subsection{Identification of the expert panel}

According to the purpose of the research subjects and the Delphi method, we established the inclusion criteria: (1) knowledgeable about nursing caring and held academic influence in caring field; (2) devoted in the field that related to humanities, nursing administration, nursing education, and hospital management for more than 10 years; (3) associate professor and above or associate director of nursing and above; (4) bachelor degree and above; (5) being able to offer comprehensive opinions; and (6) willing to participate in our survey. After initial screening, we involved a multidisciplinary panel to support the study and also balance the potential bias produced by specialists about their own field. We identified panel members through searching the database named China National Knowledge Infrastructure. In the database, when we typed key words such as "caring", relevant scholars appeared. We followed this with a combination of purposive and snowball sampling.

The multidisciplinary panel consisted of 24 experts, two medical humanities specialists, 15 nursing administrators, five nursing educators, and the last two experts engaged in hospital management. The expert panel did not contain the first-line nurse managers because we have been had a cross-sectional survey to explore nurse managers' experiences and expectation for caring training content before. There was also no first-line nurse manager who met our requirements.

\subsection{Consensus level}

The required level of consensus was defined in advance. Three necessary conditions had to be fulfilled: (1) an average score (mean) of 4 or above, (2) a consensus percentage of at least $80 \%$, and (3) a coefficient of variation of 0.2 or below. In the consensus calculation, the consensus percentage was calculated by classifying the values $1-3$ as not important (0) and 4-5 (1) as important.

\subsection{Ethical approval}

The research proposal was submitted to and approved by the ethics committee of Tongji Medical College, Huazhong University of Science and Technology (IORG No: IORG0003571). All participants were informed about the purposes and the methods of the study. Participants were anonymous and not known to each other. The record of participants' contact details and response status for each round was stored separately from their responses. Moreover, the participants were reassured that their responses would be kept confidential, the results of this survey would not be used for any purpose other than this study, and their identities would not be revealed in any research reports and publications of the study. Experts have the right of quitting at any time during the research.

\subsection{Data collection}

\subsubsection{Round 1}

The questionnaire was sent electronically to a multidisciplinary panel of 24 individuals in October 2014. Sets consisting of a written request for cooperation in the survey and a request to send back their responses within about two weeks, together with the questionnaire we introduced before. Approximately 2 weeks later, nonresponders were contacted a second time electronically. Frequency, mean, standard deviation, and coefficient of variation were calculated for each item. Content analysis was performed by researchers, and expert words and phrases were used as often as possible when constructing the round 2 survey.

\subsubsection{Round 2}

There was a one-week interval between the two rounds. In November 2014, experts who agreed to participate in the first round and sent back responses were sent a second survey. In the second round, we added some items according to the opinion of the multidisciplinary panel. Experts were only asked to rate the importance of each items. Weight would be calculated based on their score. Nonresponders were contacted a second time electronically within 1 week of the survey due date.

\section{Result}

We used e-mail to invite 24 experts for participation in the study. In all, 23 people agreed to participate, and 22 experts (a response rate of $91.7 \%$ ) from 14 provinces (Hubei, Jiangsu, Henan, Beijing, Shanghai, Guangdong, Yunnan, Sichuan, Hunan, Liaoning, Shaanxi, Shanxi, Shandong, and Fujian) completed the second round. Dropout occurred between the first round and second round among the groups of nursing administrators and females.

\subsection{Sample characteristics}

The sample included 22 experts who responded to all two rounds of the Delphi survey. The age of the 
participants ranged from 37 to 61 years, with a mean of 48.35 years. The average working time was 27.96 years (ranged from 11 to 46 years). Years of experience in management or education was 12-35, and the average was 22.4. The demographic information supported that they were experts in their own field. Other information of these experts is listed in Table 1.

\subsection{Round 1 survey results}

All the items were conformed to the selection principle and preserved, which means that these items were very important or important. Experts suggested separating the item 'Caring for Patients and Their Family Members' into two themes. They believed that some items should be increased or adjusted. Adjustment included items changed into another module and words adjusted. Finally, we added four items and adjusted six items according to the opinion from these experts.

\begin{tabular}{|c|c|c|}
\hline Item & $n$ & $\%$ \\
\hline \multicolumn{3}{|l|}{ Gender } \\
\hline Female & 20 & 90.9 \\
\hline Male & 2 & 9.1 \\
\hline \multicolumn{3}{|l|}{ Age, years } \\
\hline$<40$ & 3 & 13.6 \\
\hline $40-49$ & 8 & 36.4 \\
\hline $50-59$ & 10 & 45.5 \\
\hline$>60$ & 1 & 4.5 \\
\hline \multicolumn{3}{|l|}{ Degree } \\
\hline Bachelor & 3 & 13.6 \\
\hline Master & 14 & 63.6 \\
\hline Doctor & 5 & 22.7 \\
\hline \multicolumn{3}{|l|}{ Years of experience } \\
\hline$<20$ & 2 & 9.1 \\
\hline 20-29 & 10 & 45.5 \\
\hline 30-39 & 9 & 40.9 \\
\hline$>39$ & 1 & 4.5 \\
\hline \multicolumn{3}{|l|}{ Position } \\
\hline Professor & 7 & 31.8 \\
\hline Associate professor & 2 & 9.1 \\
\hline Director of nursing & 10 & 45.5 \\
\hline Associate director of nursing & 3 & 13.6 \\
\hline \multicolumn{3}{|l|}{ Discipline } \\
\hline Nursing Education & 6 & 27.3 \\
\hline Nursing Administration & 6 & 27.3 \\
\hline Nursing Education and Administration & 6 & 27.3 \\
\hline Medical Humanities & 2 & 9.1 \\
\hline Hospital Management & 2 & 9.1 \\
\hline
\end{tabular}

Table 1. Demographic information of experts $(n=22)$.

\subsection{Round 2 survey results}

During the second round, no suggestion had been proposed and all the 23 items met the selection principle. We used analytic hierarchy process to calculate weight of each item according to the score. Details are listed in Table 2.

\section{Discussion}

\subsection{Validity of the survey result}

In this study, we used a Delphi technique to seek consensus on the importance of different topics in the content of caring training for nurse managers. It was useful for achieving consensus in areas that were lacking empirical evidence. ${ }^{45}$ Recognized experts in nursing education, administration, medical humanities, and hospital management participated in this study. The multidisciplinary panel with different years of work experiences came from 14 provinces, which balance the potential bias produced by specialists about their own field. A high level of cooperation was obtained with respective response rates of $95.8 \%$ and $95.7 \%$ from people who received the surveys in the first and second rounds. All the experts who engaged in two rounds of survey gave their comments and suggestions, which means that they were closely involved in the survey. The Delphi result correlated with the cross-sectional study, ${ }^{44}$ indicating that the results were a valid consequence. The above results suggested that the present study achieved an adequate level of reliability and validity as a Delphi study.

\subsection{Twenty-three training items for which consent was obtained}

This study has identified 23 caring training contents belonging to four modules, which should be achieved by nurse managers. The modules were progressive and followed the logic flow. The Caring Framework for Excellence in Nursing Education was found by a review of the related literature. The Framework contained three parts: Practice, Scholarship, and Leadership. ${ }^{46}$ The presenting characteristics showed a striking resemblance to our findings. Practice corresponded to caring knowledge and caring ability. Scholarship and Research were similar. The last part, Leadership, was clearly the same. It has been stated that nursing caring education was not something that should end up with the discontiguous and sporadic. ${ }^{34}$ Our research got a systematic, scientific, feasible caring training content for nurse managers. 


\begin{tabular}{|c|c|c|c|c|c|}
\hline Module & Training content & Mean & $\mathrm{SD}$ & CV & Weight \\
\hline \multirow[t]{11}{*}{ Caring Knowledge and Caring Ability } & & 4.86 & 0.35 & 0.07 & 0.4490 \\
\hline & The caring overview & 4.45 & 0.51 & 0.11 & 0.0176 \\
\hline & Belief and spirit in caring & 4.95 & 0.21 & 0.04 & 0.0852 \\
\hline & Caring theories & 4.86 & 0.35 & 0.07 & 0.0760 \\
\hline & Human-based management & 4.59 & 0.59 & 0.13 & 0.0266 \\
\hline & Courtesy in caring & 4.55 & 0.67 & 0.15 & 0.0245 \\
\hline & Caring in communication & 4.82 & 0.50 & 0.10 & 0.0589 \\
\hline & Strategies for self-care & 4.82 & 0.40 & 0.08 & 0.0589 \\
\hline & Caring for patients & 4.81 & 0.40 & 0.08 & 0.0494 \\
\hline & Caring for family members & 4.64 & 0.49 & 0.11 & 0.0343 \\
\hline & Narrative medicine in human caring & 4.45 & 0.67 & 0.15 & 0.0176 \\
\hline \multirow[t]{7}{*}{ Caring Leadership } & & 4.55 & 0.51 & 0.11 & 0.1761 \\
\hline & Creating caring culture & 4.73 & 0.46 & 0.10 & 0.0449 \\
\hline & Organizing caring activities & 4.59 & 0.59 & 0.13 & 0.0305 \\
\hline & Caring for nursing working environment & 4.73 & 0.46 & 0.10 & 0.0449 \\
\hline & Caring responsibility for nurse mangers & 4.41 & 0.67 & 0.15 & 0.0131 \\
\hline & Caring standards, criteria, and patterns & 4.50 & 0.51 & 0.11 & 0.0213 \\
\hline & Assessing and measuring caring in nursing & 4.50 & 0.51 & 0.11 & 0.0213 \\
\hline \multirow[t]{4}{*}{ Caring Education } & & 4.68 & 0.48 & 0.10 & 0.2259 \\
\hline & Selecting education contents & 4.64 & 0.49 & 0.11 & 0.0904 \\
\hline & Teaching approaches & 4.64 & 0.49 & 0.11 & 0.0904 \\
\hline & Evaluating the training effect & 4.50 & 0.51 & 0.11 & 0.0452 \\
\hline \multirow[t]{5}{*}{ Caring Research } & & 4.36 & 0.58 & 0.13 & 0.1490 \\
\hline & Selecting topics for research & 4.45 & 0.51 & 0.11 & 0.0401 \\
\hline & Research methods in caring & 4.27 & 0.63 & 0.15 & 0.0180 \\
\hline & Article writing & 4.41 & 0.50 & 0.11 & 0.0287 \\
\hline & Evidence-based caring practice & 4.68 & 0.48 & 0.10 & 0.0621 \\
\hline
\end{tabular}

Note: SD, standard deviation; $C V$, coefficient of variation.

Table 2. Caring training contents for nurse manager

\subsubsection{Module l: Caring knowledge and caring ability}

The weight of caring knowledge and caring ability was much higher than others because this module contained 10 items. Nurse managers used knowledge and ability of caring to perform their job. ${ }^{2}$ Apparently, it was the most important and fundamental part. The first step to be an eminent nurse manger was to be a good nurse. However, there were still significant differences between nurses and nurse managers. So, this module based on caring knowledge and caring ability for a caring nurse, but not exactly the same. The caring overview helped nurse managers have a rough idea of caring. Nurse managers would devote themselves to caring when they finished learning belief and spirit in caring. Caring theories was the foundation. Human-based management, caring in communication, strategies for self-care, caring for patients, and caring for family members were the core of nursing caring. Combined with the hot topics in China, we added narrative medicine in human caring.
As China was a land of courtesy and Chinese nurses took much count of courtesy, so we confirmed courtesy in caring in caring knowledge and ability module.

\subsubsection{Module II: Caring leadership}

Nursing managers were responsible for caring in their unit. They had to translate caring to the systems level and set the stage for a caring organization. ${ }^{2}$ So they needed to create caring culture and caring environment and organize caring activities. Nurse managers were always confused about how to implement caring into clinical practice, ${ }^{2,17,18}$ so we selected the caring standards, criteria, and patterns to guide them. It was also an issue to realize the basic thing a nurse manager should do, thus, caring responsibility for nurse mangers came into view. When all items were finished, we assessed and measured it in order to make sure whether they had been promoted. 


\subsubsection{Module III: Caring education}

The learning procedure of nurses was divided into two parts: schools and hospitals. Obviously, hospital was the key part, where nurses could have their systematic, specific, and long-term learning. A nurse manager was the principal of education in hospital and also the person who was acquainted with every nurses. Hence, they should organize targeted training for individuals according to the department situation. In the process, teaching approaches and effective evaluation were two issues of crucial importance.

\subsubsection{Module IV: Caring research}

Nursing research was the backbone to improve and promote nursing work. Nurse managers played an exemplary role to other nurses. In all, $78 \%$ articles in the nursing field belong to nurse managers. ${ }^{47}$ Caring research helped nurse manager understand caring not only from the practice phase but also from theoretical height. When you select a topic for research, you should master the research methods, draft the manuscript, and finally, transform caring science into practice. The four items were the key steps in nursing research.

\section{Conclusions}

A multidisciplinary panel has identified the necessary items for nurse managers in the caring training field,

\section{References}

1. Slettebø Å, Fredriksson L, The significance of caring. Scand J Caring Sci. 2015;29:203-204.

2. Nyberg JJ. A Caring Approach in Nursing Administration. Colorado: University Press of Colorado; 2010.

3. Fleck LM. Just caring: assessing the ethical and economic costs of personalized medicine. Urol Oncol. 2014;32:202-206.

4. Arslan-Özkan İ, Okumuş H, Buldukoğlu K. A randomized controlled trial of the effects of nursing care based on Watson's Theory of Human Caring on distress, self-efficacy and adjustment in infertile women. J Adv Nurs. 2014;70:1801-1812.

5. Watson J, Brewer BB. Caring science research: criteria, evidence, and measurement. J Nurs Adm. 2015;45:235-236.

6. Foss Durant A, McDermott S, Kinney G, et al. Caring science: transforming the ethic of caringhealing practice, environment, and culture within which was the first step for caring education for nurse managers. The contents would serve as a guideline to healthcare institutions. Nurse managers can choose any module to have a system learning according to their practical needs, totally or separately.

Based on the training contents, there is still need to explore the effective teaching method and the appropriate way to measure the effect of the training. An empirical study should be conducted in the future. Additional research is required to revise the contents to make sure the integrity and integration of the content.

\section{Limitation}

There were two limitations existing in the present research: one was the selection of experts and another was the questionnaire. At present, the Chinese nursing experts in human caring are rather limited, and the academic level of Chinese nurses is lower than that of nurses from other countries, so the resource of expert selection was limited in this study, and the size of sample was also smaller than the researcher's original plan. The questionnaire, which was designed by the research team, may not contain all essential components in caring training contents and may still miss some items.

\section{Conflict of interest}

All contributing authors declare no conflicts of interest. an integrated care delivery system. Perm J. 2015;19:e136-e142.

7. Edvardsson D, Watt E, Pearce F. Patient experiences of caring and person-centredness are associated with perceived nursing care quality. $\mathrm{J} \mathrm{Adv}$ Nurs. 2017;73:217-227.

8. Lalleman PC, Smid GA, Lagerwey MD, et al. Curbing the urge to care: a Bourdieusian analysis of the effect of the caring disposition on nurse middle managers' clinical leadership in patient safety practices. Int J Nurs Stud. 2016;63:179-188.

9. Salmela S, Koskinen C, Eriksson K. Nurse leaders as managers of ethically sustainable caring cultures. J Adv Nurs. 2016;73:871-882.

10. Roch G, Dubois CA, Clarke SP. Organizational climate and hospital nurses' caring practices: a mixedmethods study. Res Nurs Health. 2014;37:229-240.

11. Morvillers JM. Care, the caring, the cure and the caregiver. Rech Soins Infirm. 2015;31:77-81. 
12. McFarland MR, Wehbe-Alamah HB. Leininger's Culture Care Diversity and Universality. Burlington: Jones \& Bartlett Learning; 2014.

13. Bishop M. Work engagement of older registered nurses: the impact of a caring-based intervention. J Nurs Manag. 2013;21:941-949.

14. Wade GH, Osgood B, Avino K, et al. Influence of organizational characteristics and caring attributes of managers on nurses' job enjoyment. J Adv Nurs. 2008;64:344-353.

15. Daley KA. Nurse leaders never stop caring - and learning. J Infus Nurs. 2014;37:13-14.

16. Tallo D. Good nursing requires caring practitioners with knowledge. Nurs Stand. 2015;29:33.

17. Richards K. The urgency of creating a culture of caring: start with you! Nurs Econ. 2016;34:152-155.

18. Stewart L, Holmes C, Usher K. Reclaiming caring in nursing leadership: a deconstruction of leadership using a Habermasian lens. Collegian. 2012;19:223-229.

19. Erdmann AL, de Andrade SR, de Mello AL, et al. Practices for caring in nursing: Brazilian research groups. Int Nurs Rev. 2011;58:379-385.

20. Vandenhouten C, Kubsch S, Peterson M, et al, Watson's theory of transpersonal caring: factors impacting nurses professional caring. Holist Nurs Pract. 2012;26:326-334.

21. Zander B, Blümel M, Busse R. Nurse migration in Europe - can expectations really be met? Combining qualitative and quantitative data from Germany and eight of its destination and source countries. Int J Nurs Stud. 2013;50:210-218.

22. Harrison S. The caring, committed majority of nurses need better support. Nurs Stand. 2013;27:12-13.

23. Peng X, Liu YL, Zeng Q. Caring behaviour perceptions from nurses of their first-line nurse managers. Scand J Caring Sci. 2015;29:708-715.

24. Cawthorne J. Even most caring nurses can end up feeling desensitised. Nurs Stand. 2016;30:32.

25. Guo Y, Shen J, Ye X, et al. The design and testing of a caring teaching model based on the theoretical framework of caring in the Chinese context: a mixedmethod study. Nurs Educ Today. 2013;33:912-918.

26. Hsu TC, Turton MA, Cheng SF, et al. Developing online continuing education content for enhancing caring among Taiwan nurses. J Contin Educ Nurs. 2013;44:186-192.

27. Salehian M, Heydari A, Aghebati N, et al. Principlebased concept analysis: caring in nursing education. Electron Physician. 2016;8:2160-2167.

28. Hills M, Watson J. Creating a Caring Science Curriculum: An Emancipatory Pedagogy for Nursing. New York: Springer Publishing Company; 2011.
29. Karen MJ. A Comparison of Levels of Empowerment and Clinical Decision-Making in Senior Bachelor of Science Nursing Students Enrolled in A Curriculum based on A Caring Nurse Theorist and A Curriculum not based on A Caring Nurse Theorist. Greeley: University of Northern Colorado; 2011.

30. Chen $Y$, Ge W, Ning XX, Zhang Q. Culturing humanistic care ability of vocational nursing students. J Nurs Sci. 2015;30:72-74 (in Chinese).

31. Jiang X, Tan X, Liu YL, Lu CH. Application of KABP mode in teaching of humanistic nursing care to nursing students. J Nurs Sci. 2016;31:81-83(in Chinese).

32. Guo HX, Chen H. Practice of setup of Concern and care course in nursing undergraduate education. Chin Nurs Res. 2010;24:2054-2055 (in Chinese).

33. Yue P, Li RY, Wang YL, Ma LL, Wu Y. Design and implementation effects of caring in nursing curriculum for undergraduate nursing students. J Nurs Admin. 2013;13:567-569 (in Chinese).

34. Brown CJ, Wilson CB. One university making a difference in graduate education: caring in the online learning environment. $J$ Holist Nurs. 2016;34:402-407.

35. Boussaid L, Dahlgren M, Lindwall L. Nurses learn caring theory by being co-researchers in a surgical setting. Nurse Educ Today. 2012;32:393-398.

36. Lamke D, Catlin A, Mason-Chadd M. "Not just a theory": the relationship between Jin Shin Jyutsu® selfcare training for nurses and stress, physical health, emotional health, and caring efficacy. J Holist Nurs. 2014;32:278-289.

37. Jiao J. Effects of Human Care Course on Nurses Caring Ability and Purpose in Life. Zhongnan University; 2012 (in Chinese).

38. Wang QH. Influence of human caring theory training program on nurses caring behavior. $J$ Nurs Sci. 2011;26:1-3(in Chinese).

39. Hsu TC, Chiang-Hanisko L, Lee-Hsieh J, et al. Effectiveness of an online caring curriculum in enhancing nurses' caring behavior. J Contin Educ Nurs. 2015;46:416-424.

40. Loraine PB. Caring in Nursing Curriculum Design: A Quantitative Study of Attitudes, Belief, and Practices of Associate Degree Nursing Faculty. Minneapolis: Capella University; 2005.

41. Herbst AM, Swengros DI, Kinney G. How to teach human caring: nurse educator role in transformational learning for a large healthcare system. J Nurses Staff Dev. 2010;26:6-11.

42. Nyberg JJ. Teaching caring to the nurse administrator. JONA. 1993;23:11-17.

43. Sitzman KL. Mindful communication for caring online. ANS Adv Nurs Sci. 2016;39:38-47. 
44. Liu YL, Chen R, Lin CJ, Li XY, Zeng TY. The caring training state and expectation of nursing manager. Chin J Med Edu. 2016;32:221-225 (in Chinese).

45. Keeney S, McKenna H, Hasson F. The Delphi Technique in Nursing and Health Research. Chichester: Wiley; 2010.
46. Sawatzky JA, Enns CL, Ashcroft TJ, et al. Teaching excellence in nursing education: a caring framework. J Prof Nurs. 2009;25:260-266.

47. Wu HY, Liu YL, Xu JJ, Yan QY. Investigation on factors affecting research fields and paper- publishing of chief nurse. J Nurs Sci. 2013;28:43-45 (in Chinese). 